\title{
Findings from the LHC/HL-LHC \\ Programme
}

\author{
Andrea Bastianin
}

\section{Contents}

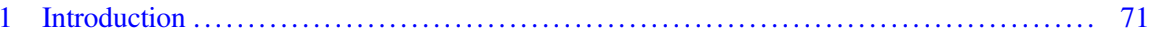

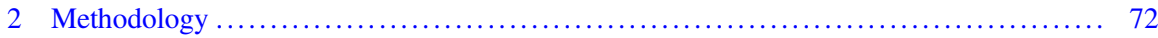

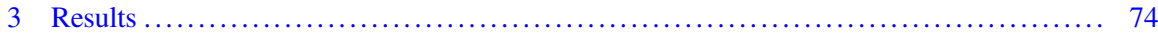

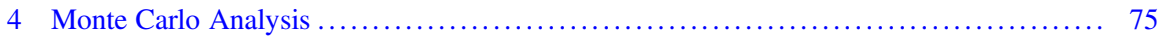

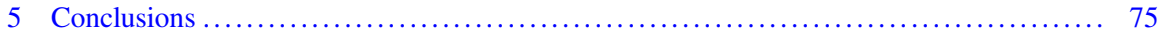

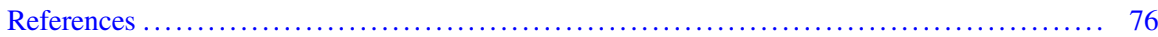

\section{Introduction}

CERN and more generally Big Science Centres (BSCs) are ideal testing grounds for theoretical and empirical economic models. In fact, the operations of BSCs generate unique data for economists (about e.g. procurement contracts, staff, students and alike, software, media coverage). See Castelnovo et al. [1] and references therein. Moreover, governance and procurement policies of BSCs are interesting topics in management studies (see e.g. [2, 3]). Furthermore, innovation and breakthrough technologies arising from BSCs are one of the drivers of long-run economic growth [4-6]. Finally yet importantly, as shown in Fig. 1, CERN and its accelerator complex represent a unique international research infrastructure (RI) that generate a variety of societal benefits that go well beyond the boundaries of the scientific community using them for research purposes. See Florio [7] and Florio and Sirtori [8] for recent overviews of the CBA of RI. Boardman et al. [9] is a useful general introduction to the methodology.

A social CBA is an appropriate methodology for evaluating a RI because it translates in quantitative terms the multi-dimensional benefits ascribed to it [10]. The social CBA machinery has found applications in several policy-relevant

\footnotetext{
A. Bastianin $(\bowtie)$

Department of Economics, Management, and Quantitative Methods (DEMM), University of Milan, Milan, Italy

e-mail: andrea.bastianin@unimi.it
} 


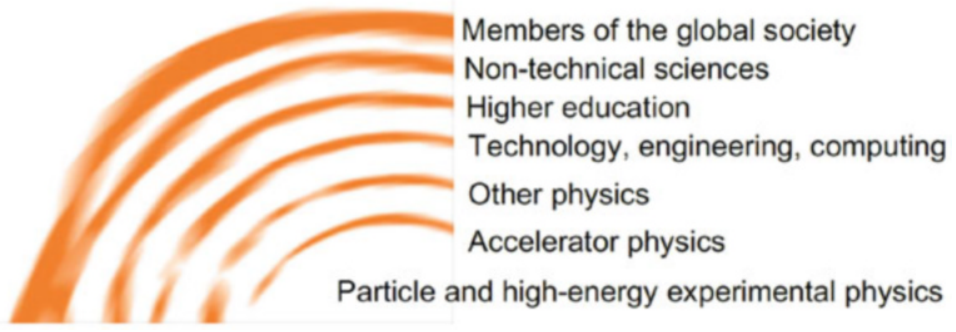

Fig. 1 The "onion" model of involvement: societal benefits of particle accelerator. Source: http:// cds.cern.ch/record/2653673

contexts. For instance, successfully passing a CBA test is required for co-financing major projects with the European Regional Development Fund and the Cohesion Fund. Similarly, the "Horizon 2020-Work Programme 2018-2020 on European research infrastructures" mentions that the preparatory phase of new ESFRI projects (www.esfri.eu) should include a CBA [11]. Assessment of the socio-economic impacts of RIs has been included in the latest edition of the "Guide to Cost-Benefit Analysis of Investment Projects" of the [12].

\section{Methodology}

One of the main object of interest in a social CBA is the estimated expected Net Present Value $(N P V)$ of a RI at the end of a defined observation period:

$$
E(N P V)=E[D B-D C]
$$

Interpretation of results is straightforward: a RI passes the CBA test when the cumulative sum of discounted social benefits $(D B)$ exceeds the cumulative sum of discounted social costs $(D C)$, that is when the expected $N P V$ is greater than zero. See Bastianin and Florio [13] for further details.

The practical implementation of model (1) involves the following steps:

1. Identification of the social benefits and costs that are relevant for the HL-LHC;

2. Estimate present and future social benefits and costs;

3. Use Monte Carlo simulations to estimate the probability distribution of social costs, benefits and of the NPV of the project.

Since the HL-LHC represents an upgrade of an existing RI - the LHC - we carry out the CBA for two scenarios. The baseline scenario is CERN with the HL upgrade and the counterfactual scenario (CFS) is the operation of the LHC until its end of life without the HL upgrade. The horizon of the analysis spans the 1993-2038 period. From 1993 to 2014, the two scenarios overlap and hence the costs and benefits are 


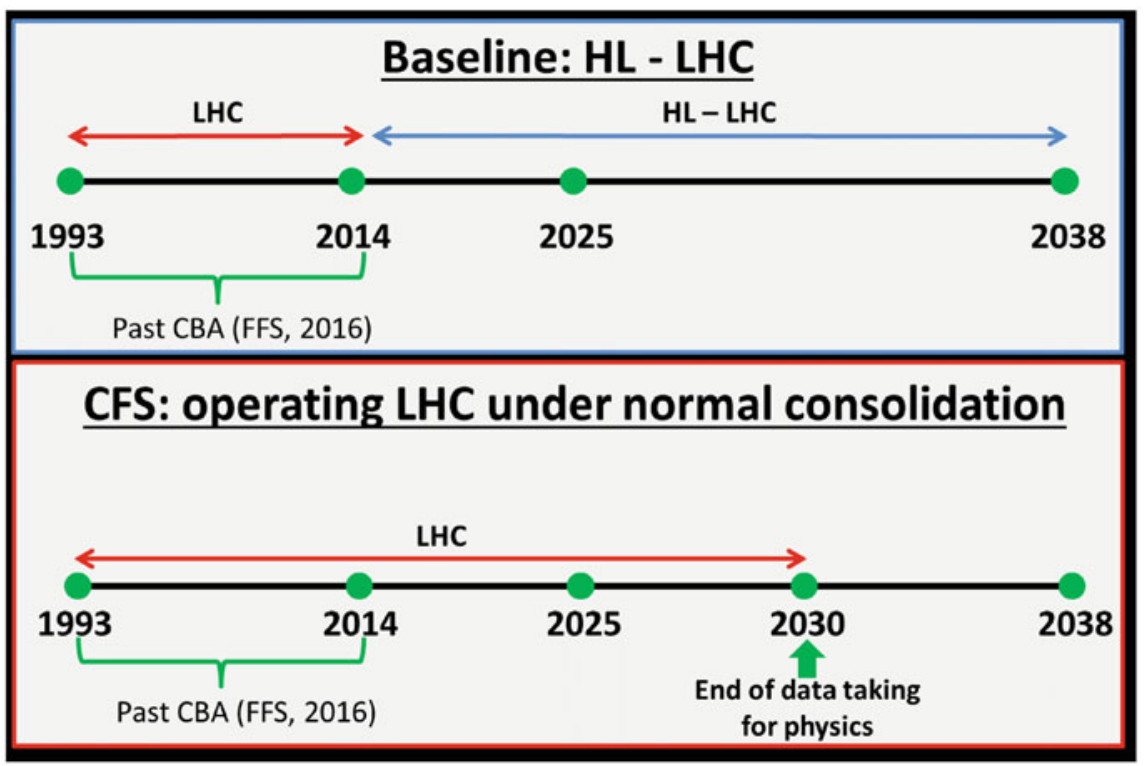

Fig. 2 HL-LHC and Counterfactual scenario: 1993-2038. Notes: dates in the figure represent time schedules that are consistent with assumptions from discussions with CERN experts

identical to those considered in the CBA of the LHC by Florio, Forte and Sirtori [14] (see Fig. 2).

In the CFS the LHC is operated "with ordinary consolidation activities"; after 2031, data taking ends and CERN staff shift their engagement to other scientific activities. After the collider is switched-off, the equipment remains in the tunnel and the underground infrastructure would be subject to appropriate monitoring and safety procedures without operating. Planned maintenance and repair activities are considered. In both scenarios we consider the following as the most relevant benefits:

1. The value of training (or human capital formation) for students and early stage researchers

2. Technological or industrial spillovers for collaborating firms and other economic agents

3. Cultural effects for the public

4. Academic publications and pre-prints for scientists

5. Existence or public good value of the RI for non-users

Notice that although the horizon of the analysis spans the years 1993-2038 some benefits extend over this time period (see Fig. 3). Non-users are people who currently do not directly use the services of the RI, but are better off simply knowing that new knowledge might be created. 


\begin{tabular}{|l|l|}
\hline arXiv.org & $\begin{array}{l}\text { Scientists (S): 1993-2063 } \\
\text { - Preprints \& publications }\end{array}$ \\
\hline & $\begin{array}{l}\text { Early Stage Researchers (H): 1993-2080 } \\
\text { - Human capital formation }\end{array}$ \\
\hline $\begin{array}{l}\text { Firms (T) : 1993-2038 } \\
\text { - Technological spillovers and free ICT }\end{array}$ & $\begin{array}{l}\text { General public (C): 1993-2038 } \\
\text { - Cultural effects of outreach }\end{array}$ \\
\hline $\begin{array}{l}\text { Taxpayers (EXV): 1993-2038 } \\
\text { - Public good value of science }\end{array}$ \\
\hline
\end{tabular}

Fig. 3 Social benefits of the HL-LHC

\section{Results}

In what follows we consider the incremental benefits produced by the HL-LHC, namely the cumulated discounted value of the benefits associated with the HL-LHC minus the corresponding cumulated discounted value arising in the CFS.

For brevity, we focus on three categories of benefits. There are two reasons for restricting our attention to these categories: First, they are quantitatively the most relevant. Second, CERN can-to some extent- "control" these benefits and therefore they represent a strategic lever for promoting future RI.

Benefits for students and early stage researchers measure the salary increase or premium over the entire work period for individuals that have been involved in the LHC program. This is the single most important benefit; in fact, it represents $40 \%$ of total incremental benefit due to the HL-LHC. Industrial spillovers arise for firms working with CERN by resulting in new products, services, creation of new business opportunities and more efficient operation for companies. Overall, benefits in this category account for $37 \%$ of the total incremental benefit generated by the HL-LHC scenario. Although we have considered a variety of cultural effects, most of the benefits in this category are due to onsite CERN visitors and visitors of CERN travelling exhibitions. Cultural benefits account for $5 \%$ of the total incremental benefit.

Comparing the benefits and costs, shows that the Net Present Value of HL-LHC is positive and greater than the one of the counterfactual scenario. The difference between the NPVs of the two scenarios is the direct benefit of the HL-LHC project, since the alternative scenario is to continue the operation of the LHC only until the end of its lifetime with no further operation. The ratio between the HL-LHC and CFS total cost difference and the HL-LHC and CFS total benefit difference is 1.7. This 
implies that every CHF spent on the HL-LHC project generates $1.7 \mathrm{CHF}$ of benefits for the society.

\section{Monte Carlo Analysis}

One key step in a CBA is the Monte Carlo analysis that relies on simulations to deliver an estimate of the uncertainty of costs and benefits of the RI. Uncertainty arises not only because of the long time horizon of the CBA, but also because in the construction of scenarios for costs and benefits we rely on a set on a set of unknown parameters that can be estimated from the data, selected from previous studies or guess-estimated with the consultation of experts. These parameters, data and proper formulas lead to an estimate of the yearly value of costs and benefits. Drawing at random these parameters from appropriate statistical distribution functions and repeating the CBA a large number of times allows to estimate the empirical distributions of social costs, benefits and of the NPV.

Application of this methodology to the HL-LHC scenario yields an estimate of the probability of obtaining a negative NPV equal to $13 \%$. It is fair to report that the CBA of the HL-LHC is based on a set of very conservative assumptions about some of the benefits that have probably led to under-estimating them.

\section{Conclusions}

In view of the application of the CBA methodology to other RI at CERN or in other BSCs it is important to stress both its merits and its limits. Results of a social CBA do not depend on the scientific utility of a RI, nor can they be used to rank different RI based on their discovery potential.

What a successful CBA does is to identify and quantify the most relevant social costs and benefits related with a RI. The attractiveness of CERN for Early Stage Researchers (ESR) is key for passing the social CBA test. Relations with firms in the supply chain, development of Information and Communication Technologies (ICT), and cultural effects-especially those related to onsite visitors-are additional strategic levers that CERN could use to boost the societal benefits. A further aspect that is crucial for the improvement of CBA is data collection during the daily operation of BSC. Better data leads to more accurate and reliable estimates of social cost and benefits. Therefore, we identify data collection as a key factor to improve the CBA of RI.

Acknowledgments The analysis underlying this note has been prepared as a contribution to the FCC study (DCC-GOV-CC-0004, EDMS 1390795) in the frame of the Collaboration Agreement between the University of Milan and CERN (KE3044/ATS). Several people at CERN have 
contributed to the analysis underlying this short note. Special thanks go to Panagiotis Charitos, Johannes Gutleber Lucio Rossi and Florian Sonneman for insightful discussion.

\section{References}

1. Castelnovo P., Florio M., Forte S., Rossi L., \& Sirtori, E. (2018). The economic impact of technological procurement for large-scale research infrastructures: Evidence from the Large Hadron Collider at CERN. Research Policy, 47(9): 1853-1867.

2. Florio M., Giffoni F. (2018). Scientific Research at CERN as a Public Good: A Survey to French Citizens. CERN-ACC-2018-0024. Available online at: https://cds.cern.ch/record/ 2635861.

3. Vuola O., Hameri A.P. (2006) Mutually benefiting joint innovation process between industry and big-science. Technovation 26(1): 3-12.

4. Breshnahant T., \& Trajtenberg M. (1995). General purpose technologies: engines of growth?" Journal of Econometrics, 65(1): 83-108

5. Crépon B., Duguet E., and Mairesse J. (1998). Research, innovation and productivity: an econometric analysis at the firm level. Economics of Innovation and New Technology, 7(2), $115-158$.

6. Helmers C. \& Overman H. G. (2017). My precious! The location and diffusion of scientific research: evidence from the Synchrotron Diamond Light Source. The Economic Journal, 127 (604), 2006-2040.

7. Florio M., (2019) Investing in Science. Social Cost-Benefit Analysis of Research Infrastructure, MIT Press.

8. Florio, M., \& Sirtori, E., (2016) "Social benefits and costs of large scale research infrastructures." Technological Forecasting and Social Change, 112, 65-78.

9. Boardman, A. E., Greenberg, D. H., Vining, A. R., \& Weimer, D. L. (2017). Cost-benefit analysis: concepts and practice. 4th Edition. Cambridge University Press.

10. Florio M., Bastianin A., Castelnovo P. (2018a). "The socio-economic impact of a breakthrough in the particle accelerators' technology: a research agenda", Nuclear Instruments and Methods in Physics Research Section A, 909, 21-26.

11. European Commission (2017). Horizon 2020 - Work Programme 2018-2020. European research infrastructures (including e-Infrastructures). Annex 4, European Commission Decision C(2017)7124 of 27 October 2017. Available online at: https://ec.europa.eu/program.

12. European Commission (2014). Guide to Cost-Benefit Analysis of Investment projects. DG Regional and Urban Policy.

13. Bastianin, A. \& Florio, M. (2018). Social Cost Benefit Analysis of HL-LHC, CERN Technical Report, CERN-ACC-2018-0014. Available online at: https://cds.cern.ch/record/2319300.

14. Florio, M., Forte, S., \& Sirtori, E., (2016) Forecasting the socio-economic impact of the Large Hadron Collider: A cost-benefit analysis to 2025 and beyond.” Technological Forecasting and Social Change, 112, 38-53.

\section{Full Presentation}

https://indico.cern.ch/event/727555/contributions/3461267/attachments/1868055/3072623/ Bastianin_Findings_from_the_LHC.pdf 
Open Access This chapter is licensed under the terms of the Creative Commons Attribution 4.0 International License (http://creativecommons.org/licenses/by/4.0/), which permits use, sharing, adaptation, distribution and reproduction in any medium or format, as long as you give appropriate credit to the original author(s) and the source, provide a link to the Creative Commons licence and indicate if changes were made.

The images or other third party material in this chapter are included in the chapter's Creative Commons licence, unless indicated otherwise in a credit line to the material. If material is not included in the chapter's Creative Commons licence and your intended use is not permitted by statutory regulation or exceeds the permitted use, you will need to obtain permission directly from the copyright holder. 